\title{
Investigation on Boron Alpha Nanotube by Studying Their M-Polynomial and Topological Indices
}

\author{
Deeba Afzal $\left(\mathbb{D},{ }^{1}\right.$ Farkhanda Afzal $\mathbb{D}^{2},{ }^{2}$ Sabir Hussain $\left(\mathbb{D},{ }^{1}\right.$ Faryal Chaudhry ${ }^{\circ},{ }^{1}$ \\ and Dhana Kumari Thapa $\left.{ }^{3}\right)^{3}$ \\ ${ }^{1}$ Department of Mathematics and Statistics, The University of Lahore, Lahore 54000, Pakistan \\ ${ }^{2}$ Department of Humanities and Basic Sciences, MCS, National University of Sciences and Technology, \\ Islamabad 44000, Pakistan \\ ${ }^{3}$ Department of Mathematics and Statistics, Padmakanya Campus, Tribhuvan University, Kathmandu, Nepal \\ Correspondence should be addressed to Dhana Kumari Thapa; dhankumari.thapa@pkmc.tu.edu.np
}

Received 7 November 2021; Accepted 28 December 2021; Published 28 January 2022

Academic Editor: Gohar Ali

Copyright (c) 2022 Deeba Afzal et al. This is an open access article distributed under the Creative Commons Attribution License, which permits unrestricted use, distribution, and reproduction in any medium, provided the original work is properly cited.

Graph theory provides an effective tool such as graph polynomial and topological indices (TIs) to the chemist to analyze the different chemical structures. TIs are the numerical entity deducted from the molecular structure. TI helps to study the relationship between the physicochemical properties and structure of the chemical compound. In this article, we investigate the boron $\alpha$-nanotube by computing its M-polynomial and then deducing its TI. Results are also shown by plotting the graphs.

\section{Introduction}

Chemical graph theory plays an important role in analysis, designing, interpreting, modeling, and understanding chemical substances. The molecular graph is composed of vertices (atoms) and edges (chemical bonds). Chemical graph theory has many applications during the study of chemical substances $[1,2]$. The chemical graph theory provides different tools for mathematical modeling of molecular structure. This modeling is useful for the analysis of chemical compounds. The analysis of chemical compounds is made conceivable by using topological indices (TIs). A large number of TIs are introduced and applied to study pharmacology and theoretical chemistry $[3,4]$.

The first TI which correlated with the boiling points of alkanes was introduced by $\mathrm{H}$. Wiener called the Wiener index in 1947 [5]. Until now, thousands of indices are designed and used in chemical graph theory [6]. A degreedependent topological index for the graph $G$ is defined as follows:

$$
I(G)=\sum_{e=x y \in E_{G}} f\left(d_{x}, d_{y}\right)
$$

Equation (1) is rewritten by counting the same enddegree edges in chemical graph as follows:

$$
I(G)=\sum_{j \leq k} m_{j k} f(j, k),
$$

where $\left\{d_{x}, d_{y}\right\}=\{j, k\}$ and the total number of edges $x y$ is denoted by $m_{j k}$. Some important TIs are described in [7]. Reduced reciprocal Randić index [8] is defined as $\operatorname{RRR}(G)=\sum_{x y \in E_{G}} \sqrt{\left(d_{x}-1\right)\left(d_{y}-1\right)}$. The first arithmeticgeometric index [9] is defined as $\mathrm{AG}_{1}(G)=\sum_{x y \in E_{G}}$ $d_{x}+d_{y} / 2 \sqrt{d_{x} \cdot d_{y}}$. SK indices [10] are defined as $\operatorname{SK}(G)=\sum_{x y \in E_{G}} d_{x}+d_{y} / 2, \quad \mathrm{SK}_{1}(G)=\sum_{x y \in E_{G}} d_{x} \cdot d_{y} / 2$, and $\operatorname{SK}_{2}(G)=\sum_{x y \in E_{G}}\left(d_{x}+d_{y} / 2\right)^{2}$. The first Zagreb index in terms of edge degree [11] is defined as $\operatorname{EM}_{1}(G)=\sum_{x y \in E_{G}}\left(d_{x}+d_{y}-2\right)^{2}$. General sum connectivity 
index [12] is described as $\operatorname{SCI}(G)=\sum_{x y \in E_{G}} 1 / \sqrt{d_{x}+d_{y}}$, $\operatorname{SCI}_{\lambda}(G)=\sum_{x y \in E_{G}}\left(d_{x}+d_{y}{ }^{\lambda}\right)$. Redefined third Zagreb index [13] is commuted as $\operatorname{ReZG}_{3}(G)=\sum_{x y \in E_{G}}\left(d_{x} d_{y}\left(d_{x}+d_{y}\right)\right)$.

Graph polynomials are the best way to represent a chemical graph in chemical graph theory. In this paper, we study the M-polynomial to analyze the chemical structure. M-polynomial gives the TIs by applying some derivatives and integration operations [7, 14]. In 2015, M-polynomial is introduced in [15]. TIs via M-polynomial is extensively calculated by many researchers nowadays [14, 16, 17]. M-polynomial for the graph $G$ is defined as follows:

$$
M_{G}(u, v)=\sum_{\psi \leq j \leq k \leq \Psi} m_{j k} u^{j} v^{k} .
$$

Here, $\psi=\min \left\{d_{x} \mid x \in V_{G}\right\}$ and $\Psi=\max \left\{d_{x} \mid x \in V_{G}\right\}$. Closed form via M-polynomial of TI is mentioned in Table 1.

The operators used are defined as follows:

$$
\begin{aligned}
& D_{v}^{1 / 2} M_{G}(u, v)=\sqrt{v \frac{\partial}{\partial v} M_{G}(u, v)} \cdot \sqrt{M_{G}(u, v)}, D_{v} M_{G}(u, v)=v \frac{\partial}{\partial v} M_{G}(u, v), \\
& D_{u}^{1 / 2} M_{G}(u, v)=\sqrt{u \frac{\partial}{\partial u} M_{G}(u, v)} \cdot \sqrt{M_{G}(u, v)}, D_{u} M_{G}(u, v)=u \frac{\partial}{\partial u} M_{G}(u, v), \\
& S_{v}^{1 / 2} M_{G}(u, v)=\sqrt{\int_{0}^{v} \frac{M_{G}(u, t)}{t} \mathrm{~d} t} \cdot \sqrt{M_{G}(u, v)}, \mathrm{JM}_{G}(u, v)=M_{G}(u, u), \\
& S_{u}^{1 / 2} M_{G}(u, v)=\sqrt{\int_{0}^{u} \frac{M_{G}(t, v)}{t} \mathrm{~d} t} \cdot \sqrt{M_{G}(u, v)}, Q_{u(\alpha)} M_{G}(u, v)=u^{\alpha} M_{G}(u, v) .
\end{aligned}
$$

\section{Chemical Graph of Boron $\alpha$-Nanotube}

The search for small size, low cost, and high efficient materials is the most intersecting topic nowadays. To get this goal, there is a need to study the chemical and physical behavior of chemical substances. So, nanotechnology becomes the most important field in the twenty-first century. By using the chemical graph theory technique, nanostructures are transformed into a mathematical model and then inspected under numerous parameters. Due to attractive features such as work function, transport property, electronic structure, and structural stability, boron $\alpha$-nanotubes have gained an important place in modern times $[18,19]$. The boron $\alpha$-nanotube is constructed by boron $\alpha$-nanosheet consisting of $q$ column and $l$ rows. There are three methods to connecting the first and last column of boron $\alpha$-nanosheet: armchair, zigzag, and chiral [20].

On the basis of rows, there are two types of boron $\alpha$-nanotube such as $l \equiv 0(\bmod 3)$ shown in Figure $1(\mathrm{a})$ and $l \equiv 2(\bmod 3)$ shown in Figure $1(\mathrm{~b})$. In the present study, we consider the armchair connecting of boron $\alpha$-sheet to form a boron $\alpha$-nanotube for $l \equiv 0(\bmod 3)$ and symbolize as $B_{\alpha} \mathrm{NT}_{l q}$ shown in Figure 2. Vertex partition of $B_{\alpha} N T_{l q}$ is presented in Table 2, and edge partition is in Table 3.

\section{M-Polynomial and Topological Indices of Boron $\alpha$-Nanotube}

Theorem 1. If $B_{\alpha} N T_{l q}$ represents a boron $\alpha$-nanotube, then M-polynomial of $B_{\alpha} N T_{l q}$ is $M_{B_{\alpha} N T_{l q}}(u, v)=q / 2 u^{3} v^{3}+$ $q u^{3} v^{5}+q u^{3} v^{6}+3 q / 2 u^{4} v^{4}+2 q u^{4} v^{5}+q u^{4} v^{6}+q / 2(3 l-$ 8) $u^{5} v^{5}+q(2 l-8) u^{5} v^{6}[21,22]$.

Theorem 2. Let $B_{\alpha} N T_{l q}$ represent the boron $\alpha$-nanotube and $M_{B_{\alpha} N T_{l q}}(u, v)=q / 2 u^{3} v^{3}+q u^{3} v^{5}+q u^{3} v^{6}+3 q / 2 u^{4} v^{4}+2 q u^{4}$ $v^{5}+q u^{4} v^{6}+q / 2(3 l-8) u^{5} v^{5}+q(2 l-8) u^{5} v^{6}$. Then,

(1) $R R R\left[B_{\alpha} N T_{l q}\right]=(6+4 \sqrt{5}) q l-1 / 2(21-4 \sqrt{2}$ $-8 \sqrt{3}+20 \sqrt{5}-2 \sqrt{10}-2 \sqrt{15}) q$

(2) $A G_{1}\left[B_{\alpha} N T_{l q}\right]=1 / 30(45+11 \sqrt{30}) q l+1 / 60(45 \sqrt{2}+$ $54 \sqrt{5}+25 \sqrt{6}+16 \sqrt{12}-55 \sqrt{30}-120) q$

(3) $S K\left[B_{\alpha} N T_{l q}\right]=37 / 2 q l-35 / 2 q$

(4) $S K_{1}\left[B_{\alpha} N T_{l q}\right]=195 / 4 q l-249 / 4 q$

(5) $S K_{2}\left[B_{\alpha} N T_{l q}\right]=98 q l-121 q$

(6) $E M_{1}\left[B_{\alpha} N T_{l q}\right]=258 q l-352 q$

(7) $\operatorname{SCI}\left[B_{\alpha} N T_{l q}\right]=1 / 220(33 \sqrt{10}+40 \sqrt{11}) q l+1 / 1320$ $(1320+825 \sqrt{2}+110 \sqrt{6}-396 \sqrt{10}-600 \sqrt{11}) q$ 
TABLe 1: Topological indices derived from $\mathbf{M}_{\mathbf{G}}(\mathbf{u}, \mathbf{v})$.

\begin{tabular}{|c|c|c|}
\hline Topological index & & Derivation from $\mathbf{M}_{\mathbf{G}}(\mathbf{u}, \mathbf{v})$ \\
\hline$\overline{R R R}[G]$ & $=$ & $\left.D_{u}^{1 / 2} D_{v}^{1 / 2} Q_{u(-1)} Q_{v(-1)} M_{G}(u, v)\right|_{u=v=1}$ \\
\hline $\mathrm{AG}_{1}[G]$ & $=$ & $1 /\left.2 D_{u} J S_{u}^{1 / 2} S_{v}^{1 / 2} M_{G}(u, v)\right|_{u=1}$ \\
\hline SK $[G]$ & $=$ & $1 /\left.2\left(D_{u}+D_{v}\right) M_{G}(u, v)\right|_{u=v=1}$ \\
\hline $\mathrm{SK}_{1}[G]$ & $=$ & $1 /\left.2 D_{u} D_{v} M_{G}(u, v)\right|_{u=v=1}$ \\
\hline $\mathrm{Sk}_{2}[G]$ & $=$ & $1 /\left.4 D_{u}^{2} J \mathrm{M}_{G}(u, v)\right|_{u=1}$ \\
\hline $\mathrm{EM}_{1}[G]$ & $=$ & $\left.D_{u}^{2} Q_{u(-2)} J_{G}(u, v)\right|_{u=1}$ \\
\hline $\operatorname{SCI}[G]$ & $=$ & $\left.S_{u}^{1 / 2} \operatorname{JM}_{G}(u, v)\right|_{u=1}$ \\
\hline $\operatorname{SCI}_{\lambda}[G]$ & $=$ & $\left.D_{u}^{\lambda} \mathrm{JM}_{G}(u, v)\right|_{u=1}$ \\
\hline $\operatorname{ReZG}_{3}[G]$ & $=$ & $\left.D_{u} D_{v}\left(D_{u}^{u}+D_{v}\right) M_{G}(u, v)\right|_{u=v=1}$ \\
\hline
\end{tabular}
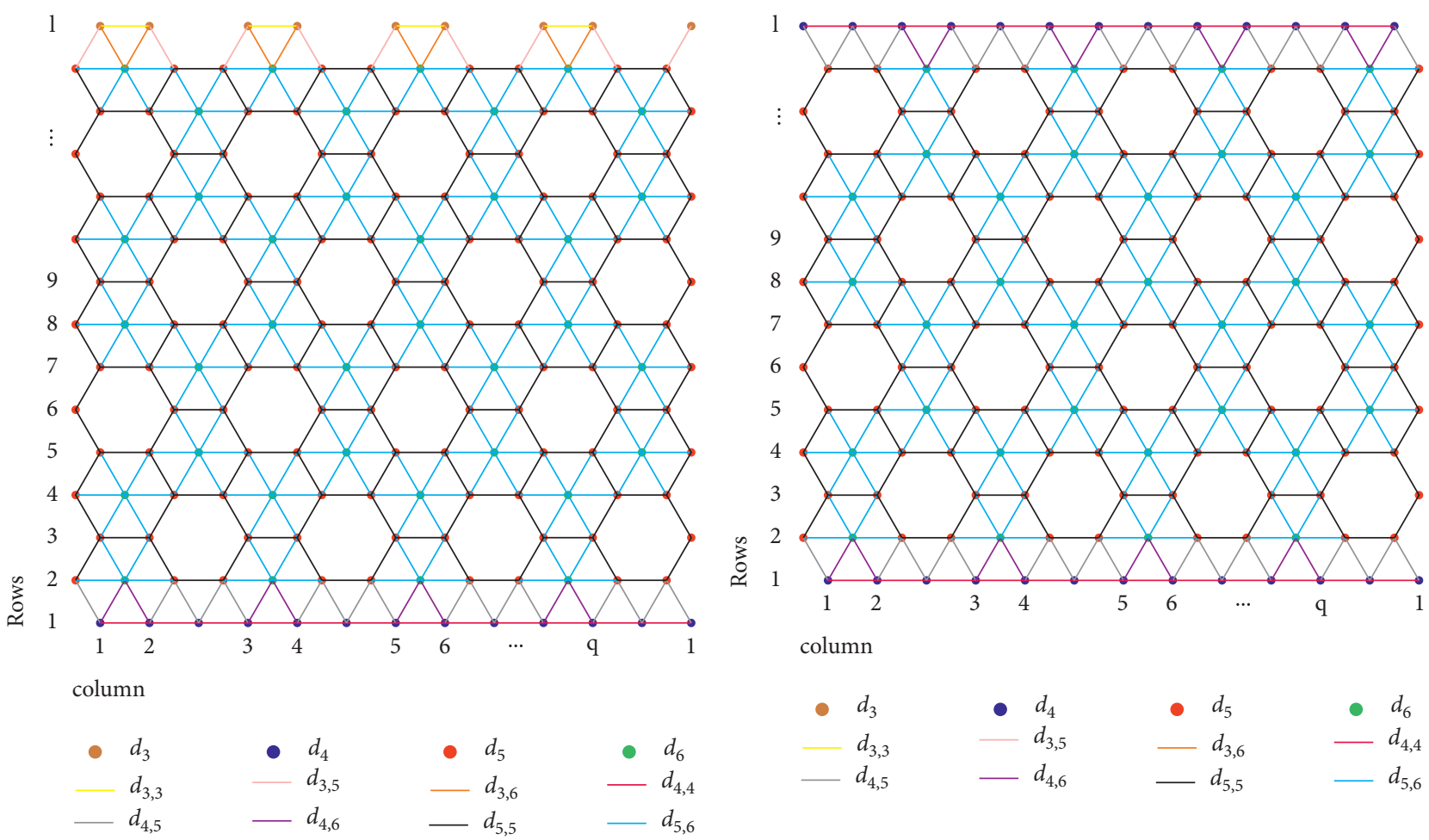

(a)

column

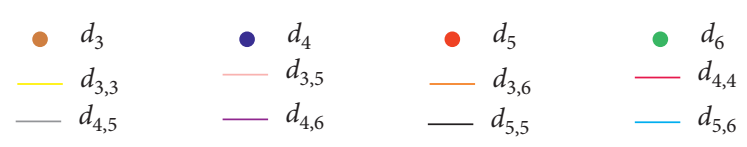

(b)

FIgURE 1: Boron $\alpha$-nanosheet $B_{\alpha} N S_{l q}$ : (a) $l=0(\bmod 3)$; (b) $l=2(\bmod 3)$.

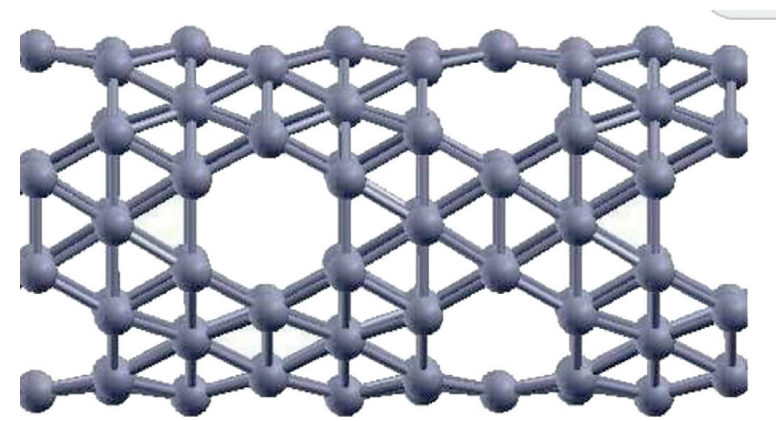

FIGURE 2: Boron $\alpha$-nanotube $\left(\mathbf{B}_{\alpha} \mathbf{N T}_{\mathbf{l q}}\right)$.

TABLE 2: Vertex partition of boron $\alpha$-nanotube $\left(\mathbf{B}_{\alpha} \mathbf{N T}_{\mathbf{l q}}\right)$.

\begin{tabular}{llcccc}
\hline $\mathbf{d}_{\mathbf{x}}$ & 3 & 4 & 5 & 6 & Total vertices \\
\hline Number of vertices & $q$ & $3 / 2 q$ & $q(l-2)$ & $1 / 6 q(2 l-3)$ & $4 / 3 q 1$ \\
\hline
\end{tabular}


TABLE 3: Edge partition of boron $\alpha$-nanotube $\left(\mathbf{B}_{\alpha} \mathbf{N T}_{\mathbf{l q}}\right)$.

\begin{tabular}{lc}
\hline$\left(\mathbf{d}_{\mathbf{x}}, \mathbf{d}_{\mathbf{y}}\right)$ & Number of edges \\
\hline$(3,3)$ & $1 / 2 q$ \\
$(3,5)$ & $q$ \\
$(3,6)$ & $q$ \\
$(4,4)$ & $3 / 2 q$ \\
$(4,5)$ & $2 q$ \\
$(4,6)$ & $q$ \\
$(5,5)$ & $1 / 2 q(3 l-8)$ \\
$(5,6)$ & $q(2 l-5)$ \\
Total edges & $1 / 2 \mathbf{q}(7 \mathbf{1}-4)$ \\
\hline
\end{tabular}

(8) $\operatorname{SCI}_{\lambda}\left[B_{\alpha} N T_{l q}\right]=1 / 2\left(3 \cdot 10^{\lambda}+4 \cdot 11^{\lambda}\right) q l+1 / 2\left(6^{\lambda}+\right.$ Proof.

$\left.5 \cdot 8^{\lambda}+6 \cdot 9^{\lambda^{\lambda}}-6 \cdot 10^{\lambda}-10 \cdot 11^{\lambda}\right) q$

(9) $\operatorname{ReZG}_{3}\left[B_{\alpha} N T_{l q}\right]=1035 q l-1549 q$

$$
\begin{aligned}
& Q_{v(-1)} M_{B_{\alpha} N T_{l q}}(u, v)=\frac{q}{2} u^{3} v^{2}+\mathrm{qu}^{3} v^{4}+\mathrm{qu}^{3} v^{5}+\frac{3 q}{2} u^{4} v^{3}+2 \mathrm{qu} u^{4} v^{4}+\mathrm{qu}^{4} v^{5} \\
& +\frac{q}{2}(3 l-8) u^{5} v^{4}+q(2 l-8) u^{5} v^{5}, \\
& Q_{u(-1)} Q_{v(-1)} M_{B_{\alpha} N T_{l q}}(u, v)=\frac{q}{2} u^{2} v^{2}+\mathrm{qu}^{2} v^{4}+\mathrm{qu}^{2} v^{5}+\frac{3 q}{2} u^{3} v^{3}+2 q u^{3} v^{4}+q u^{3} v^{5} \\
& +\frac{q}{2}(3 l-8) u^{4} v^{4}+q(2 l-8) u^{4} v^{5}, \\
& D_{v}^{1 / 2} Q_{u(-1)} Q_{v(-1)} M_{B_{\alpha} N T_{l q}}(u, v)=\frac{\sqrt{2}}{2} \mathrm{qu}^{2} v^{2}+2 \mathrm{qu}^{2} v^{4}+\sqrt{5} \mathrm{qu}^{2} v^{5}+\frac{3 \sqrt{3}}{2} \mathrm{qu}^{3} v^{3}+4 \mathrm{qu}^{3} v^{4} \\
& +\sqrt{5} q u^{3} v^{5}+q(3 l-8) u^{4} v^{4}+\sqrt{5} q(2 l-8) u^{4} v^{5}, \\
& D_{u}^{1 / 2} D_{v}^{1 / 2} Q_{u(-1)} Q_{v(-1)} M_{B_{\alpha} N T_{l q}}(u, v)=\mathrm{qu}^{2} v^{2}+2 \sqrt{2} \mathrm{qu}^{2} v^{4}+\sqrt{10} \mathrm{qu}^{2} v^{5}+\frac{9}{2} \mathrm{qu}^{3} v^{3} \\
& +4 \sqrt{3} q u^{3} v^{4}+\sqrt{15} q u^{3} v^{5}+2 q(3 l-8) u^{4} v^{4}+2 \sqrt{5} q(2 l-8) u^{4} v^{5}, \\
& S_{v}^{1 / 2} M_{B_{\alpha} N T_{l q}}(u, v)=\frac{\sqrt{3}}{6} \mathrm{qu}^{3} v^{3}+\frac{\sqrt{5}}{5} \mathrm{qu}^{3} v^{5}+\frac{\sqrt{6}}{6} \mathrm{qu}^{3} v^{6}+\frac{3}{4} \mathrm{qu}^{4} v^{4}+\frac{2 \sqrt{5}}{5} \mathrm{qu}^{4} v^{5} \\
& +\frac{\sqrt{6}}{6} q u^{4} v^{6}+\frac{\sqrt{5}}{10} q(3 l-8) u^{5} v^{5}+\frac{\sqrt{6}}{6} q(2 l-8) u^{5} v^{6} \text {, } \\
& S_{u}^{1 / 2} S_{v}^{1 / 2} M_{B_{\alpha} N T_{l q}}(u, v)=\frac{1}{6} \mathrm{qu}^{3} v^{3}+\frac{\sqrt{15}}{15} \mathrm{qu}^{3} v^{5}+\frac{\sqrt{2}}{6} \mathrm{qu}^{3} v^{6}+\frac{3}{8} \mathrm{qu}^{4} v^{4}+\frac{\sqrt{5}}{5} \mathrm{qu}^{4} v^{5} \\
& +\frac{\sqrt{6}}{12} q u^{4} v^{6}+\frac{1}{10} q(3 l-8) u^{5} v^{5}+\frac{\sqrt{30}}{30} q(2 l-8) u^{5} v^{6}, \\
& J S_{u}^{1 / 2} S_{v}^{1 / 2} M_{B_{\alpha} N T_{l q}}(u, v)=\frac{1}{6} \mathrm{qu}^{6}+\frac{1}{120}(45+8 \sqrt{15}) \mathrm{qu}^{8}+\frac{1}{30}(5 \sqrt{2}+6 \sqrt{5}) \mathrm{qu}^{9} \\
& +\frac{\sqrt{6}}{12} \mathrm{qu}^{10}+\frac{1}{10} q(3 l-8) u^{10}+\frac{\sqrt{30}}{30} q(2 l-8) u^{11}, \\
& D_{u} J S_{u}^{1 / 2} S_{v}^{1 / 2} M_{B_{\alpha} N T_{l q}}(u, v)=q u^{6}+\frac{1}{15}(45+8 \sqrt{15}) \mathrm{qu}^{8}+\frac{3}{10}(5 \sqrt{2}+6 \sqrt{5}) \mathrm{qu}^{9} \\
& +\frac{5 \sqrt{6}}{6} \mathrm{qu}^{10}+q(3 l-8) u^{10}+\frac{11 \sqrt{30}}{30} q(2 l-8) u^{11}, \\
& \frac{1}{2} D_{u} \mathrm{JS}_{u}^{1 / 2} S_{v}^{1 / 2} M_{B_{\alpha} N T_{l q}}(u, v)=\frac{1}{2} \mathrm{qu}^{6}+\frac{1}{30}(45+8 \sqrt{15}) \mathrm{qu}^{8}+\frac{3}{20}(5 \sqrt{2}+6 \sqrt{5}) \mathrm{qu}^{9} \\
& +\frac{5 \sqrt{6}}{12} \mathrm{qu}^{10}+\frac{1}{2} q(3 l-8) u^{10}+\frac{11 \sqrt{30}}{60} q(2 l-8) u^{11},
\end{aligned}
$$




$$
\begin{aligned}
& D_{u} M_{B_{\alpha} N T_{l q}}(u, v)=\frac{3}{2} q u^{3} v^{3}+3 q u^{3} v^{5}+3 q u^{3} v^{6}+6 q u^{4} v^{4}+8 q u^{4} v^{5} \\
& +4 q u^{4} v^{6}+\frac{5}{2} q(3 l-8) u^{5} v^{5}+5 q(2 l-8) u^{5} v^{6} \\
& D_{v} M_{B_{\alpha} N T_{l q}}(u, v)=\frac{3}{2} \mathrm{qu}^{3} v^{3}+5 q u^{3} v^{5}+6 q u^{3} v^{6}+6 q u^{4} v^{4}+10 q u^{4} v^{5} \\
& +6 q u^{4} v^{6}+\frac{5}{2} q(3 l-8) u^{5} v^{5}+6 q(2 l-8) u^{5} v^{6} \\
& \left(D_{u}+D_{v}\right) M_{B_{\alpha} N T_{l q}}(u, v)=3 q u^{3} v^{3}+8 q u^{3} v^{5}+9 q u^{3} v^{6}+12 q u^{4} v^{4}+18 q u^{4} v^{5} \\
& +10 q u^{4} v^{6}+5 q(3 l-8) u^{5} v^{5}+11 q(2 l-8) u^{5} v^{6} \\
& \frac{1}{2}\left(D_{u}+D_{v}\right) M_{B_{\alpha} N T_{l q}}(u, v)=\frac{3}{2} \mathrm{qu}^{3} v^{3}+4 \mathrm{qu}^{3} v^{5}+\frac{9}{2} \mathrm{qu}^{3} v^{6}+6 \mathrm{qu} v^{4} v^{4}+9 \mathrm{qu} u^{4} v^{5} \\
& +5 q u^{4} v^{6}+\frac{5}{2} q(3 l-8) u^{5} v^{5}+\frac{11}{2} q(2 l-8) u^{5} v^{6} \\
& D_{u} D_{v} M_{B_{\alpha} N T_{l q}}(u, v)=\frac{9}{2} \mathrm{qu}^{3} v^{3}+15 q u^{3} v^{5}+18 q u^{3} v^{6}+24 q u^{4} v^{4}+40 q u^{4} v^{5} \\
& +24 q u^{4} v^{6}+\frac{25}{2} q(3 l-8) u^{5} v^{5}+30 q(2 l-8) u^{5} v^{6}, \\
& \frac{1}{2}\left(D_{u} D_{v}\right) M_{B_{\alpha} N T_{l q}}(u, v)=\frac{9}{4} \mathrm{qu}^{3} v^{3}+\frac{15}{2} \mathrm{qu}^{3} v^{5}+9 \mathrm{qu}^{3} v^{6}+12 q u^{4} v^{4}+20 q u^{4} v^{5} \\
& +12 q u^{4} v^{6}+\frac{25}{4} q(3 l-8) u^{5} v^{5}+15 q(2 l-8) u^{5} v^{6} \\
& \mathrm{JM}_{B_{\alpha} N T_{l q}}(u, v)=\frac{q}{2} u^{6}+\frac{5}{2} \mathrm{qu}^{8}+3 \mathrm{qu}^{9}+\frac{q}{2}(3 l-6) u^{10}+q(2 l-8) u^{11}, \\
& D_{u}^{2} \mathrm{JM}_{B_{\alpha} N T_{l q}}(u, v)=18 \mathrm{qu}^{6}+160 \mathrm{qu}^{8}+243 \mathrm{qu}^{9}+50 q(3 l-6) u^{10} \\
& +121 q(2 l-8) u^{11} \\
& \frac{1}{4} D_{i}^{2} \mathrm{JM}_{B_{\alpha} N T_{l q}}(u, v)=\frac{9}{2} \mathrm{qu}^{6}+40 \mathrm{qu}^{8}+\frac{243}{4} \mathrm{qu}^{9}+\frac{25}{2} q(3 l-6) u^{10} \\
& +\frac{121}{4} q(2 l-8) u^{11} \\
& Q_{u(-2)} \mathrm{JM}_{B_{\alpha} N T_{l q}}(u, v)=\frac{q}{2} u^{4}+\frac{5}{2} \mathrm{qu}^{6}+3 \mathrm{qu}^{7}+\frac{q}{2}(3 l-6) u^{8}+q(2 l-8) u^{9}, \\
& D_{u}^{2} Q_{u(-2)} J_{B_{\alpha} N T_{l q}}(u, v)=8 q u^{4}+90 q u^{6}+147 q u^{7}+32 q(3 l-6) u^{8}+81 q(2 l-8) u^{9}, \\
& S_{u}^{1 / 2} \mathrm{JM}_{B_{\alpha} N T_{l q}}(u, v)=\frac{\sqrt{6}}{12} \mathrm{qu}^{6}+\frac{5 \sqrt{2}}{8} \mathrm{qu}^{8}+\mathrm{qu}^{9}+\frac{3 \sqrt{10}}{10} q(3 l-6) u^{10}+\frac{\sqrt{11}}{11} q(2 l-8) u^{11}, \\
& D_{u}^{\lambda} \operatorname{JM}_{B_{\alpha} N T_{l q}}(u, v)=\frac{6^{\lambda}}{2} \mathrm{qu}^{6}+\frac{5 \cdot 8^{\lambda}}{2} \mathrm{qu}^{8}+3 \cdot 9^{\lambda} \mathrm{qu}^{9}+\frac{10^{\lambda}}{2} q(3 l-6) u^{10} \\
& +11^{\lambda} q(2 l-8) u^{11} \\
& D_{v}\left(D_{u}+D_{v}\right) M_{B_{\alpha} N T_{l q}}(u, v)=9 \mathrm{qu}^{3} v^{3}+40 q u^{3} v^{5}+54 q u^{3} v^{6}+48 q u^{4} v^{4}+90 q u^{4} v^{5} \\
& +60 \mathrm{qu}^{4} v^{6}+25(3 l-8) u^{5} v^{5}+66 q(2 l-8) u^{5} v^{6}, \\
& D_{u} D_{v}\left(D_{u}+D_{v}\right) M_{B_{\alpha} N T_{l q}}(u, v)=27 \mathrm{qu}^{3} v^{3}+120 \mathrm{qu}^{3} v^{5}+162 \mathrm{qu}^{3} v^{6}+192 \mathrm{qu}^{4} v^{4} \\
& +360 q u^{4} v^{5}+240 q u^{4} v^{6}+125(3 l-8) u^{5} v^{5}+330 q(2 l-8) u^{5} v^{6} \text {. }
\end{aligned}
$$

(1) $\operatorname{RRR}\left[B_{\alpha} \mathrm{NT}_{l q}\right]=D_{u}^{1 / 2} D_{v}^{1 / 2} Q_{u(-1)} Q_{v(-1)} M_{B_{\alpha} N T_{l q}}$ $\left.(u, v)\right|_{u=v=1}, \quad \operatorname{RRR}\left[B_{\alpha} \mathrm{NT}_{l q}\right]=(6+4 \sqrt{5}) \mathrm{ql}-1 / 2(21-$ $4 \sqrt{2}-8 \sqrt{3}+20 \sqrt{5}-2 \sqrt{10}-2 \sqrt{15}) q$.
(2) $A G_{1}\left[B_{\alpha} N T_{l q}\right]=1 /\left.2 D_{u} \mathrm{JS}_{u}^{1 / 2} S_{v}^{1 / 2} M_{B_{\alpha} N T_{l q}}(u, v)\right|_{u=1}$, $\mathrm{AG}_{1}\left[B_{\alpha} \mathrm{NT}_{l q}\right]=1 / 30(45+11 \sqrt{30}) \mathrm{ql}+1 / 60(45 \sqrt{2}$ $+54 \sqrt{5}+25 \sqrt{6}+16 \sqrt{12}-55 \sqrt{30}-120) q$. 


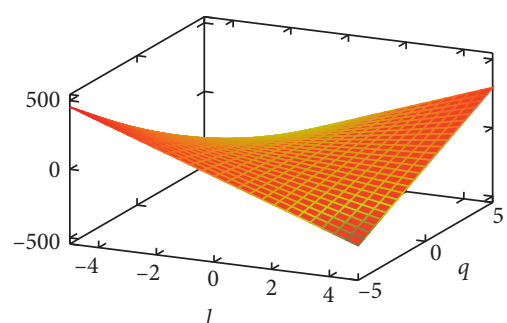

(a)

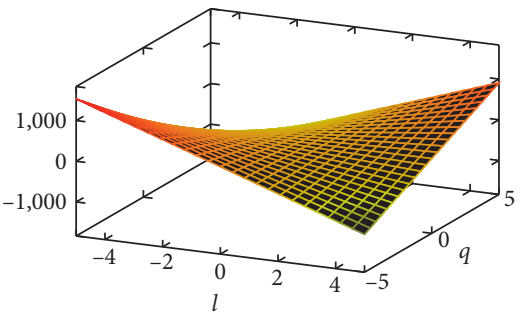

(d)

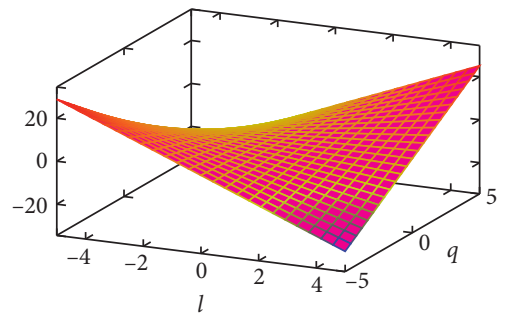

$(\mathrm{g})$

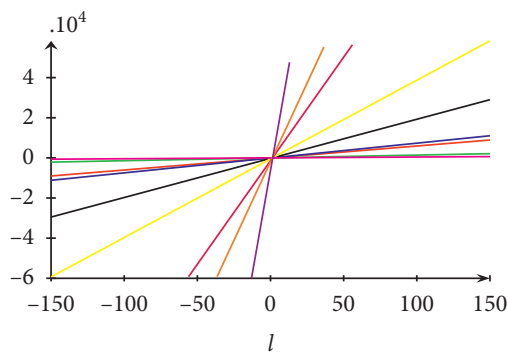

(j)

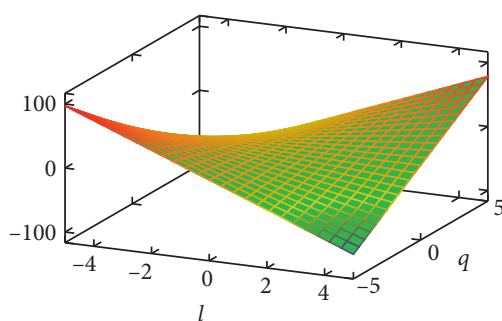

(b)

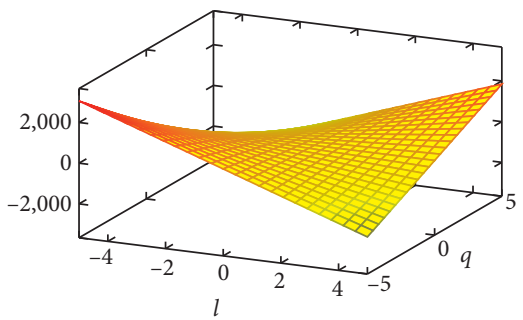

(e)

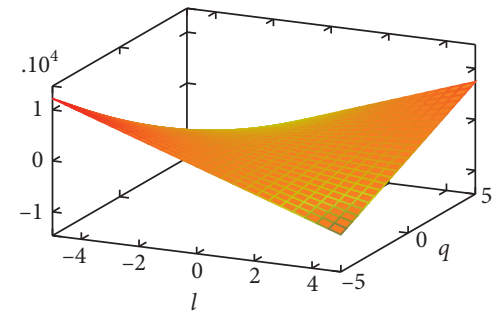

(h)

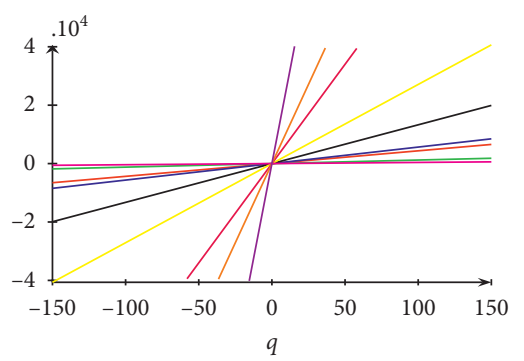

(k)

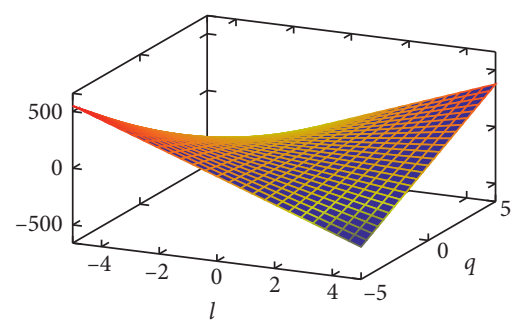

(c)

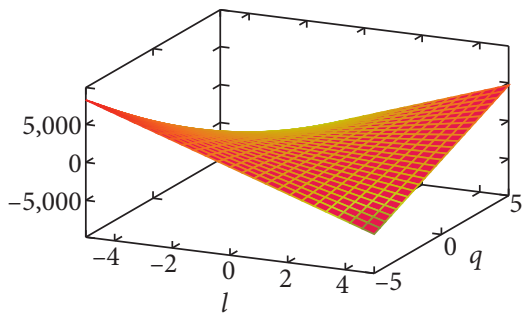

(f)

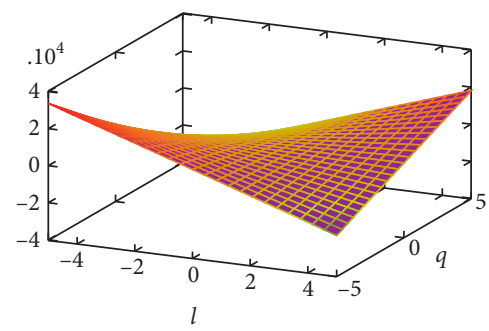

(i)

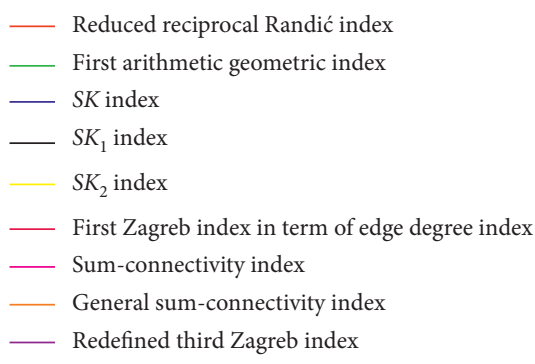

(l)

FIgURE 3: Plot of topological indices of boron $\alpha$-nanotube: (a) reduced reciprocal Randić index; (b) arithmetic-geometric index; (c) SK index; (d) $\mathrm{SK}_{1}$ index; (e) $\mathrm{SK}_{2}$ index; (f) edge version of first Zagreb index; (g) sum connectivity index; (h) general sum connectivity index; (i) redefined third Zagreb index; (j) TI of $\left[B_{\alpha} \mathrm{NT}_{l q}\right] \mid q=4$; (k) TI of $\left[B_{\alpha} \mathrm{NT}_{l q}\right] \mid l=4$; (l) colour of TI.

(3) $\operatorname{SK}\left[B_{\alpha} \mathrm{NT}_{l q}\right]=1 /\left.2\left(D_{u}+D_{v}\right) M_{B_{\alpha} N T_{l q}}(u, v)\right|_{u=v=1}=37$ $/ 2 \mathrm{ql}-35 / 2 q$.

(4) $\mathrm{SK}_{1}\left[B_{\alpha} \mathrm{NT}_{l q}\right]=1 /\left.2 D_{u} D_{v} M_{B_{\alpha} N T_{l q}}(u, v)\right|_{u=v=1}=195$ /4ql-249/4q.

(5) $\mathrm{Sk}_{2}\left[B_{\alpha} \mathrm{NT}_{l q}\right]=1 /\left.4 D_{u}^{2} \mathrm{JM}_{B_{\alpha} N T_{l q}}(u, v)\right|_{u=1}=98 \mathrm{ql}-121 q$.

(6) $\operatorname{EM}_{1}\left[B_{\alpha} \mathrm{NT}_{l q}\right]=\left.D_{u}^{2} Q_{u(-2)} \operatorname{JM}_{B_{\alpha} N T_{l q}}(u, v)\right|_{u=1}=258 \mathrm{ql}$ $-352 q$.

(7) $\mathrm{SCI}\left[B_{\alpha} \mathrm{NT}_{l q}\right]=\left.S_{u}^{1 / 2} \mathrm{JM}_{B_{\alpha} N T_{l q}}(u, v)\right|_{u=1}, \mathrm{SCI}\left[B_{\alpha} \mathrm{NT}_{l q}\right]=$ $1 / 220(33 \sqrt{10}+40 \sqrt{11}) \mathrm{ql}+1 / 1320(1320+$ $825 \sqrt{2}+110 \sqrt{6}-396 \sqrt{10}-600 \sqrt{11}) q$.
(8) $\mathrm{SCI}_{\lambda}\left[B_{\alpha} \mathrm{NT}_{l q}\right]=\left.D_{u}^{\lambda} \mathrm{JM}_{B_{\alpha} N T_{l q}}(u, v)\right|_{u=1}, \mathrm{SCI}_{\lambda}\left[B_{\alpha} \mathrm{NT}_{l q}\right]$ $=1 / 2\left(3 \cdot 10^{\lambda}+4 \cdot 11^{\lambda}\right) \mathrm{q} 1+1 / 2\left(6^{\lambda}+5 \cdot 8^{\lambda}+6 \cdot 9^{\lambda}-6 \cdot 10^{\lambda}\right.$ $\left.-10 \cdot 11^{\lambda}\right) q$.

(9) $\operatorname{ReZG}_{3}\left[B_{\alpha} \mathrm{NT}_{l q}\right]=D_{u} D_{v}\left(D_{u}+D_{v}\right) M_{B_{\alpha} N T_{l q}} \quad(u$, $v)\left.\right|_{u=v=1}=1035 q 1-1549 q$.

\section{Conclusion}

We studied the important nanotube known as boron $\alpha$-nanotube by computing their M-polynomial and then recovered some important degree-based TIs. The graphical representations of the results are presented 
in Figure 3. The results obtained will be useful in helping to solve many problems in the field of chemical analysis.

\section{Data Availability}

No data were used to support this research.

\section{Conflicts of Interest}

The author(s) declare that there are no conflicts of interest regarding the publication of this paper.

\section{References}

[1] S. C. Basak, V. R. Magnuson, G. J. Niemi, R. R. Regal, and G. D. Veith, "Topological indices: their nature, mutual relatedness, and applications," Mathematical Modelling, vol. 8, pp. 300-305, 1987.

[2] K. Burch, "Chemical applications of graph theory," in Mathematical Physics in Theoretical Chemistry, Elsevier, Amsterdam, Netherlands, 2019.

[3] E. Estrada and D. Bonchev, "Chemical graph theory," in Discrete Mathematics and its ApplicationsChapman and Hall/ CRC, Boca Raton, FL, USA, 2013.

[4] N. Trinajsti, Chemical Graph Theory, Routledge, New York, NY, USA, 1992.

[5] H. Wiener, "Structural determination of paraffin boiling points," Journal of the American Chemical Society, vol. 69, no. 1, pp. 17-20, 1947.

[6] R. Todeschini and V. Consonni, Handbook of Molecular Descriptors, Wiley, Hoboken, NJ, USA, 2000.

[7] D. Y. Shin, S. Hussain, F. Afzal, C. Park, D. Afzal, and M. R. Farahani, "Closed formulas for some new degree based topological descriptors using M-polynomial and boron triangular nanotube," Frontiers of Chemistry, vol. 8, Article ID 613873, 2021.

[8] I. Gutman, B. Furtula, and C. Elphick, "Three new/old vertexdegree-based topological indices," Communications in Mathematical and in Computer Chemistry, vol. 72, no. 3, pp. 617-632, 2014.

[9] V. Shigehalli and R. Kanabur, "Arithmetic-geometric indices of path graph," A Journal of Computer and Mathematical Sciences, vol. 6, no. 1, pp. 19-24, 2015.

[10] V. Shigehalli and R. Kanabur, "Computing degree based topological indices of polyhex nanotube," Journal of Mathematical Nanoscience, vol. 6, no. 1, pp. 47-55, 2016.

[11] A. Miličcević, S. Nikolić, and N. Trinajstić, "On reformulated Zagreb indices,” Molecular Diversity, vol. 8, no. 1, pp. 393399, 2004.

[12] Z. Du, B. Zhou, and N. Trinajstić, "On the general sumconnectivity index of trees," Applied Mathematics Letters, vol. 24, no. 3, pp. 402-405, 2011.

[13] P. Ranjini, V. Lokesha, and A. Usha, "Relation between phenylene and hexagonal squeeze using harmonic index," International Journal of Graph Theory, vol. 1, no. 4, pp. 116-121, 2013.

[14] Rajpoot and L. Selvaganesh, "Extension of M-polynomial and degree based topological indices for nanotube," TWMS Journal of Applied and Engineering Mathematics, vol. 11, pp. 268-279, 2021.

[15] E. Deutsch and S. Klawzar, "M-polynomial and degree-based topological indices," Iranian Journal of Mathematical Chemistry, vol. 6, no. 2, pp. 93-102, 2015.
[16] F. Yu, H. Iqbal, H. Iqbal, S. Munir, and J. B. Liu, "M-polynomial and topological indices of some transformed networks," AIMS mathematics, vol. 6, no. 12, pp. 13887-13906, 2021.

[17] X.-L. Wang, J.-B. Liu, M. Ahmad, M. Kamran Siddiqui, M. Hussain, and M. Saeed, "Molecular properties of symmetrical networks using topological polynomials," Open Chemistry, vol. 17, no. 1, pp. 849-864, 2019.

[18] J.-B. Liu, H. Shaker, I. Nadeem, and M. Hussain, "Topological aspects of boron nanotubes," Advances in Materials Science and Engineering, vol. 2018, Article ID 5729291, 11 pages, 2018.

[19] P. Menuel, "Computational aspects of carbon and boron nanotubes," Molecules, vol. 15, no. 12, pp. 8709-8722, 2010.

[20] V. Bezugly, J. Kunstmann, B. Grundkötter-Stock et al., "Highly conductive boron nanotubes: transport properties, work functions, and structural stabilities," ACS Nano, vol. 5, no. 6, pp. 4997-5005, 2011.

[21] D. Afzal, S. Hussain, M. Aldemir, M. Farahani, and F. Afzal, "New degree-based topological descriptors via m-polynomial of boron $\alpha$-nanotube," Eurasian Chemical Communications, vol. 2, no. 11, pp. 1117-1125, 2020.

[22] Y. C. Kwun, M. Munir, W. Nazeer, S. Rafique, and S. M. Kang, "Computational analysis of topological indices of two boron nanotubes,” Scientific Reports, vol. 8, pp. 14843-14915, 2018. 